\title{
Weibull Decision Support Systems in Maintenance
}

\author{
Khalid Aboura ${ }^{1}$, Johnson I. Agbinya², Ali Eskandarian ${ }^{3}$
}

\author{
${ }^{1}$ University of Dammam, College of Business Administration, Dammam, Saudi Arabia; kaboura@ud.edu.sa \\ ${ }^{2}$ La Trobe University, Faculty of Science, Technology and Engineering School of Engineering and Mathematical \\ Sciences, Department of Electronic Engineering, Melbourne, Australia; J.Agbinya@latrobe.edu.au \\ ${ }^{3}$ George Washington University, Department of Physics, 805 21st street, NW, suite 301,Washington D.C., U.S.A.; \\ ea1102@gwu.edu
}

\begin{abstract}
Background: The Weibull distribution is one of the most important lifetime distributions in applied statistics. Weibull analysis is the leading method in the world for fitting and analyzing lifetime data. We discuss one of the earliest decision support system for the assessment of a distribution for the parameters of the Weibull reliability model using expert information. We then present a different approach to assess the parameters distribution.

Objectives: The studies mentioned in this paper aimed to construct a distribution of the parameters of the Weibull reliability model and apply it in the domain of Maintenance Optimization.

Method: The parameters of the Weibull reliability model are considered random variables and a distribution for the parameters is assessed using informed judgment in the form of reliability estimates from vendor information, engineering knowledge or experience in the field.

Results: The results are the development of modern maintenance optimization models that can be embodied in decision support systems.

Conclusion: While the information management part is important in the building of maintenance optimization decision systems, the accuracy of the mathematical and statistical algorithms determines the level of success of the maintenance solution.
\end{abstract}

Keywords: Weibull Distribution, Reliability, Inference, Maintenance, Expert Opinion

\section{Introduction}

In maintenance optimization, a decision support system is a computer-based information system for the scheduling of events such as inspection, preventive maintenance, repair and replacement of operating equipment in manufacturing and industrial environments. The decisions are based on optimality criteria and involve the use of mathematical algorithms. The management of information is important and involves the collection of historical data. The second essential part is the set of mathematical and statistical algorithms used in the determination of optimal courses of actions. The accuracy and efficiency of these algorithms determines the level of success of the maintenance optimization routines. We present solutions to a mathematical computational problem and a statistical modeling problem needed in the solving of maintenance optimization problems. We focus on the Weibull distribution in Reliability and reintroduce one of the earliest Weibull Decision Support Systems in Reliability that incorporates expert opinion. We follow with the modern construction of a prior probability distribution for the parameters of the lifetime distribution for use in several maintenance optimization scenarios. In one of the maintenance problems, we point to the exact calculation of the renewal function in the case of an adaptive block replacement strategy. We illustrate the approaches with simulated results.

Received: $4^{\text {th }}$ November 2013; revised: $16^{\text {th }}$ January 2014; accepted $9^{\text {th }}$ February 2014 


\subsection{The Weibull distribution for lifetime data}

The Weibull distribution is one of the most important lifetime distributions in applied statistics. First identified by Fréchet (1927) and first applied by Rosin and Rammler (1933), it was described by Waloddi Weibull in 1937 and published for the first time in 1939 (Weibull, 1939) with the title 'A statistical theory of the strength of material.' It was written in order to explain the, at that time, well known but unexplained facts that the relative strength of a specimen decreases with increasing dimensions and that its bending strength is larger than its tensile strength (Weibull, 1981). Delayed by the Second World War and after an unsuccessful attempt to publish the result in a well-known British journal that deemed it to be interesting but of no practical importance, Weibull published his landmark paper in 1951 titled 'A statistical distribution function of wide applicability' (Weibull, 1951). Even then, the reaction to his paper in the 1950s was negative, varying from skepticism to outright rejection. Weibull's claim that the data could select the distribution and fit the parameters seemed too good to be true. However, pioneers in the field like Dorian Shainin and Leonard Johnson applied and improved the technique. The U.S. Air Force recognized the merit of Weibull's method and funded his research until 1975. Today, Weibull analysis is the leading method in the world for fitting and analyzing life data (Abernethy, 2006). For more than half a century the Weibull distribution has been used by statisticians in various fields and the research is ongoing (Sultan and Mahmoud, 2007; Pak et al., 2013). Together with the normal, exponential, $\chi^{2}, \mathrm{t}$ and $\mathrm{F}$ distributions, the Weibull distribution is, without any doubt, the most popular model in modern statistics (Rinne, 2009). It is particularly true in Reliability and survival analysis, where the distribution is applied to the modelling of lifetime data. The Weibull is a useful failure model in both biomedical and engineering applications (Singpurwalla, 2006).

\subsection{Reliability Assessment Incorporating Expert Opinion}

In classical statistics, in order to fit a statistical model to a life data set, the analyst estimates the parameters of the lifetime distribution that most closely fits the data. The parameters control the scale, shape and location of the distribution function (ReliaSoft Corporation, 2014). Several methods have been devised to estimate the parameters. These include probability plotting, rank regression on $\mathrm{x}$ (RRX), rank regression on $\mathrm{y}$ (RRY) and maximum likelihood estimation (MLE). In Bayesian statistics, the approach is different. The parameters of the model are considered random variables and a distribution for the parameters is assessed. We consider the problem of the assessment of a distribution for the parameters of the Weibull reliability model. Using informed judgment in the form of reliability estimates from vendor information, engineering knowledge or experience in the

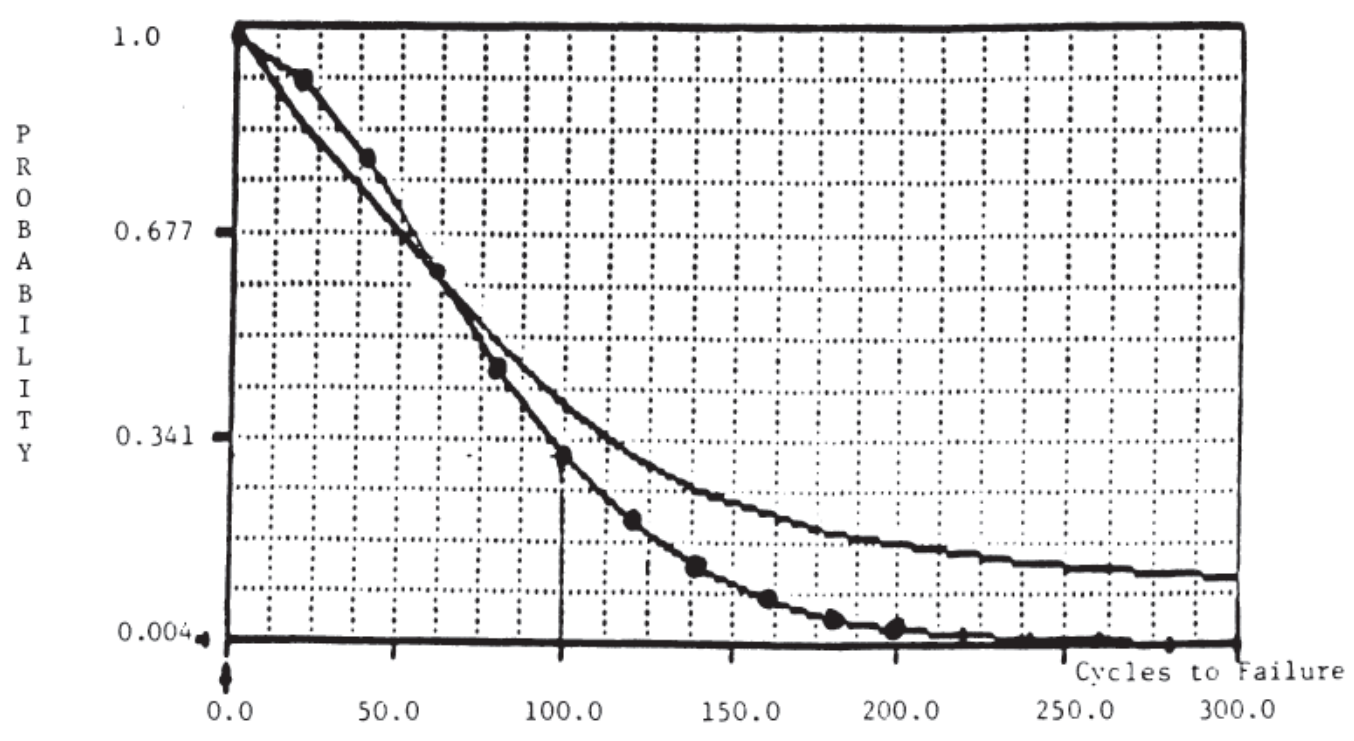

Plots of Prior (-) and Posterior (๑-) Reliability Functions for the Ball-Bearing Data. 
field, we look at building a probability distribution for the parameters $(\lambda, \beta)$ of the Weibull distribution with reliability function $R(t / \lambda, \beta)=e^{\left(-\lambda t^{\beta}\right)}$. Singpurwalla (1988) outlined the principles and discussed the development of such procedure for reliability assessment of items whose life lengths are described by the Weibull distribution, whose reliability function is shown in Figure 1. The software, An Interactive PC-Based Procedure for Reliability Assessment (IPRA), prepared by the first author of this article and cited in Goel (1988), is one of the earliest Weibull Decision Support Systems in Reliability that incorporates expert opinion. At the time, the graphics were very basic (Figure 2) and the program was still transported on a floppy disk. Aboura, Singpurwalla and Soyer (1989a, 1989b, 1989c) describe the software and the theory. Aboura and Soyer (1986) and Aboura and Campodonico (1992) provide users' manuals.

Singpurwalla and Song (1986) also presented an approach for the analysis of Weibull lifetime data using expert opinion. While many authors in the Bayesian literature use expert opinion on the values of the parameter vector (see Bousquet, 2006), Singpurwalla (1988) and Sinpurwalla and Song (1988) introduced a slightly different approach and assumed that an expert was able to provide information about the median lifetime and give an estimate of its prior mean. Fixing the marginal prior distribution of the shape parameter $\beta$, they obtained a complete prior on $(\lambda, \beta)$. For a literature review on the use of expert opinion in probabilistic risk analysis see Ouchi (2004).

\subsection{Estimation using initial reliability estimates}

There are two main difficulties using the Weibull distribution (Bousquet, 2010). First, its only conjugate prior distribution is continuous-discrete (Soland, 1969) and remains difficult to justify in real problems (Kaminskiy and Krivtsov, 2005). Second, the meanings of the scale parameter and the shape parameter greatly differ. Their values and correlation remain hard to assess by non-statistician experts (Bousquet, 2010). To alleviate this difficulty, and following on the idea of asking expert quantile information, Aboura (1995) introduced a prior elicitation procedure which uses expert opinion on the reliability of the item rather than on the parameters directly. In concept, Aboura (1995) can be viewed as an extension of the use of the median by Singpurwalla (1988) and Sinpurwalla and Song (1988). However they differ in the construction of the expert model and the resulting priors

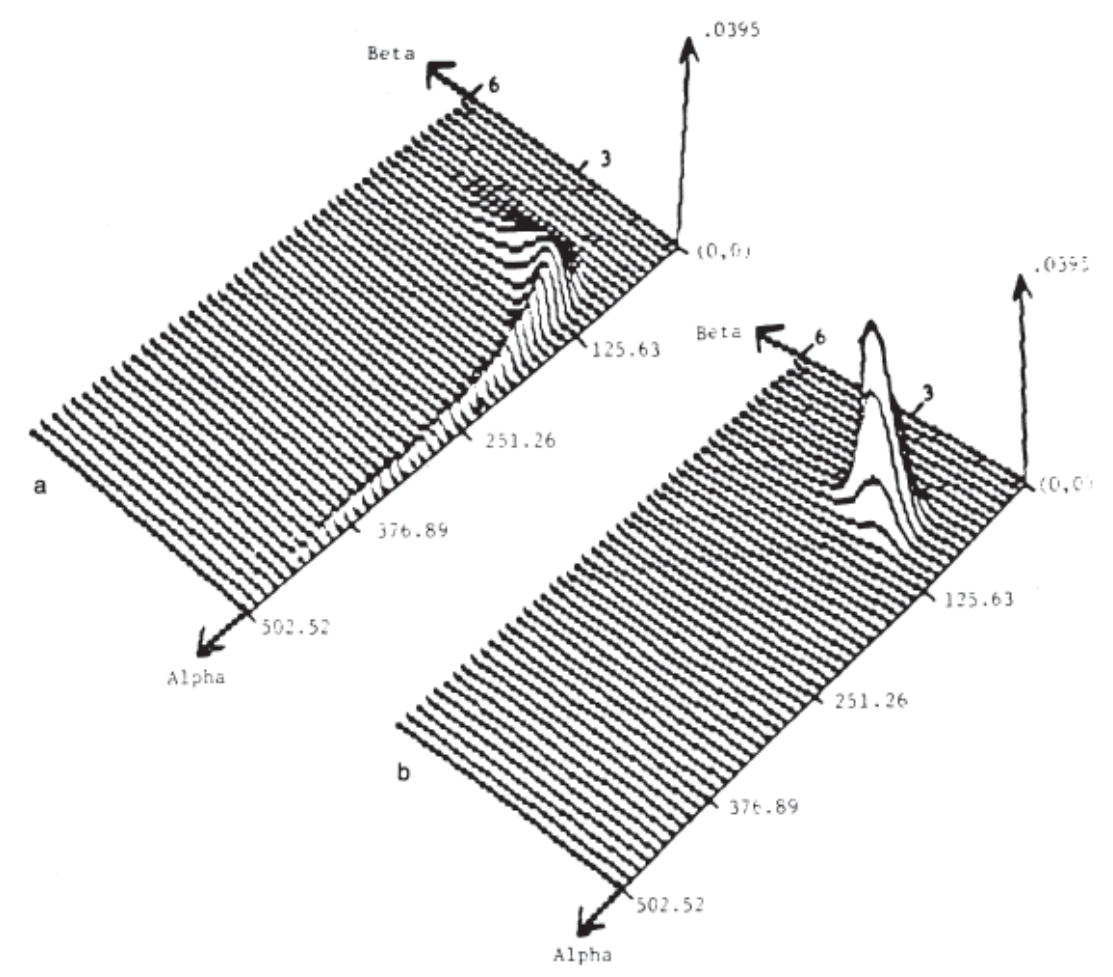

A View of the (a) Joint Prior and (b) Joint Posterior Densities of $\alpha$ and $\beta$ for the Ball-Bearing Data.

Figure 2: 3D plots in the IPRA software (Singpurwalla, 1988) 
for the Weibull parameters. In doing so, it also extends a result by Mazzuchi and Soyer (1996) who used Soland's distribution (Soland 1969) for the parameters of the Weibull lifetime distribution. The approach of Aboura (1995) is used in Aboura and Agbinya (2013) and Aboura and Robinson (2013) in the context of maintenance optimization.

\section{Research Methods in Maintenance Optimization}

The purpose of the reliability procedures discussed in the previous section is often their incorporation in maintenance optimization decision support systems. While the information management part is important in building maintenance optimization decision support systems, the accuracy of the mathematical and statistical algorithms determines the level of success of the maintenance software. In their maintenance optimization solution, Mazzuchi and Soyer (1996) used Soland's (1969) distribution for the parameters of the Weibull lifetime distribution. A discretized Beta distribution is used for the parameter $\beta$. Although such a use of Soland's distribution does provide a starting prior joint density, one could dispute the feasibility of collecting any direct information about $\beta$ from an expert, the abstract model parameter $\beta$ not having any physical meaning. One can also argue about the arbitrariness used by Mazzuchi and Soyer (1996) to select the range of the discretized Beta distribution for $\beta$, unless this range is made to cover most of the likely values of $\beta$. Aboura and Agbinya (2013) and Aboura and Robinson (2013) remedy to these shortfalls by constructing a prior density for $(\lambda, \beta)$ using estimates of observables. The range of $\beta$ and the dependence structure of $(\lambda, \beta)$ result naturally from an initial reliability estimation. The distribution of Soland (1969) is extended to include dependence and fitted through moments to the prior distribution.

\subsection{Maintenance optimization procedure using initial reliability estimates}

Upon the introduction of new equipment or at the start of a study, reliability estimates are often available in the form of vendor information or informed judgment from maintenance operators. As failure and survival data are collected, a better assessment of the life length characteristics of the items becomes possible, allowing a more effective estimation procedure. Consider a structure of $M$ identical items operating independently of each other under similar conditions. At prescribed points in time $T_{1}, T_{2}, \ldots$, etc., all items are replaced by new ones. An item that fails before the next replacement time remains failed. We let $T_{0}$ be time 0 . As failures accumulate between the replacement times, two types of data collection are possible; (1) the exact failure times are recorded (complete data) and (2) the numbers of failures per time interval are recorded (interval censored data). Aboura and Agbinya (2013) treat both cases and consider only the case of the numbers of failures between replacement times in case (2). That is the interval censored data consist of the number of failures in $\left[T_{i-1}, T_{i}\right), i=1,2$, ...The more general case of interval censored data involves inspection points in $\left[T_{i-1}, T_{i}\right)$ where the numbers of failures are recorded between the inspection points. The extension to the inspection case is straightforward given that the inspection times are fixed. A further extension would be to consider the inspection times as decision variables in the setting of an optimal maintenance strategy. Let $T$ be the lifetime of the item under consideration. We assume that reliability estimates $r^{(n)}=\left(r_{1}, r_{2}, \ldots, r_{\mathrm{n}}\right)$ are provided for different mission times $t_{i}, i=1,2, \ldots, n$, with, $r_{\mathrm{n}}+1$ being a lower bound on the reliability of the item. Assuming a Weibull model for the lifetime $T$ with reliability function, the prior distribution of $(\lambda, \beta)$ is constructed in Aboura and Agbinya (2013) and the maintenance optimization solutions provided. For example, in the Penalty Cost Model I of Aboura and Agbinya (2013), the model assumes an increasing cost function $c_{f}(j)$ for the failure of $j$ items. Various functions may be chosen to model $c_{f}$ (j) depending on the application. The maintenance optimization problem at time at time $T_{i-1}$ is

$$
\min _{\Delta T_{i}>0} \frac{c_{p}+\sum_{j=0}^{M} c_{f}(j) \operatorname{Prob}\left(N_{i}=j / D_{i-1}\right)}{\Delta T_{i}}
$$

where

$$
\begin{aligned}
\operatorname{Prob} & \left(N_{i}=j / D_{i-1}\right) \\
& =\sum_{\beta} \int_{\lambda}\left(\begin{array}{c}
M \\
j
\end{array}\right)\left(1-e^{-\lambda \Delta T_{i}^{\beta}}\right)^{j}\left(e^{-\lambda \Delta T_{i}^{\beta}}\right)^{M-j} g\left(\lambda, \beta / r^{(n)}\right) d \lambda .
\end{aligned}
$$

$g\left(\lambda, \beta / r^{(n)}\right)$ is the prior distribution constructed with the expert opinion using the initial reliability estimates. It can be shown that for an appropriate choice of the penalty function $c_{f}$, an optimal solution $\Delta T_{i}$ obtains for each stage $i=1,2$, $\ldots$, etc.

\subsection{Maintenance optimization procedure using the renewal function}

In Aboura and Robinson (2013), the maintenance scenario differs and requires the computations of the renewal function for the Weibull distribution. The maintenance optimization procedure consists of determining at each planned replacement time, $T_{i-1}$ the next preventive replacement time $T_{i}$, $i=1,2, \ldots$ In between the prescribed times $T_{1}, T_{2}, \ldots$, replacement by a new item is made upon failure of the operating item/system. The maintenance procedure applies the well-known Block Replacement protocol in which the item under consideration is replaced at predetermined points in time by a new item (or by an item repaired and brought back to the 'new' state), regardless of the age of the failed 
item. In the traditional Block Replacement approach, the time intervals between planned replacements are equal and determined at the start of the operations. In the approach by Aboura and Robinson (2013), the replacement times are determined only a stage ahead. The adaptive nature of the policy introduced reduces considerably the loss due to a Block Replacement protocol, as it reaches for an optimal replacement time. At time $T_{i-1}, i=1,2, \ldots, \Delta T_{i}=T_{i}-T_{i-1}$, is determined as the solution of

$$
\min _{\Delta T_{i}>0} \frac{c_{p}+c_{f} M\left(\Delta T_{i} / D_{i-1}\right)}{\Delta T_{i}}
$$

$c_{p}$ is the replacement cost, $c_{f}$ is the cost per failure and $D_{i-1}=\left\{f^{(m)}, s^{(l)}\right\} \cup D_{0} \cdot f^{(m)}=\left(f_{1}, f_{2}, \ldots f_{m}\right)$ and $s^{(l)}=\left(s_{1}, s_{2}, \ldots\right.$ $\left.s_{l}\right)$ are the failure and survival times respectively, observed in $\left(T_{0}, T_{i-1}\right] . D_{0}$ is the set of all relevant information known prior to and at time $T_{0}$ In this case, $D_{0}=\left\{r^{(n)}, t^{(n)}, b\right\}$ (see Aboura and Robinson, 2013). $M\left(\Delta T_{i} / D_{i-1}\right)$ is obtained by averaging over $(\lambda, \beta)$,

$$
M\left(\Delta T_{i} / D_{i-1}\right)=\sum_{j=1}^{k} \int_{0}^{\infty} M\left(\Delta T_{i} / \lambda, \beta_{j}\right) p\left(\lambda, \beta_{j} / D_{i-1}\right) d \lambda
$$

where $p\left(\lambda, \beta_{j} / D_{i-1}\right)$ is the prior distribution of $(\lambda, \beta)$ for $i=$ 1 and the posterior distribution of $(\lambda, \beta)$ at time $T_{i-1}$ for $i>$ 1 , derived from the same prior distribution at time $T_{0}$ used in Aboura and Agbinya (2013).

To solve the maintenance optimization problem, the renewal function for the Weibull distribution must be computed accurately. Let $N(t)$ be the number of failures (and renewals) in the time interval $(0, t]$, then the renewal function, $H(t)=E(N(t))$ is the expected number of renewals in that time interval. The expected number of renewals between successive times $T_{i}$ and $T_{i-1}$ is defined by, where $\Delta\left(T_{i}\right)=T_{i}-T_{i-1}$ is the optimal time interval to be determined. For the Weibull lifetime model with distribution function $F(t)=1-e^{-\lambda t^{\beta}}$, the renewal function $H(t / \lambda, \beta)$ is available in series form (Smith and Leadbetter, 1963) but becomes impracticable to calculate for most $\mathrm{t}>1, \beta>1$. One can use a simple approximation due to Smeitink and Dekker (1990), but Aboura and Robinson (2013) provide a more accurate solution, due to Constantine and Robinson (1997), for computing the Weibull renewal function and its derivative $h(t / \lambda, \beta)=d H(t / \lambda, \beta) / d t$. Another method due to Robinson (1997) can also be used to compute the renewal function to any desired degree of accuracy. That method solves directly the integral equation expression for $\mathrm{H}(\mathrm{t} / \lambda$, $\beta)$ (see e.g. Cox (1962) for general renewal theory) in terms of multi time-segment Chebyshev polynomial series. It is a method suitable for a wide range of probability density functions, both parametric and nonparametric. However, the quicker method of Constantine and Robinson (1997), dedicated to the Weibull renewal function is used to provide accurate results. The methodology can be extended with similar accuracy to the case of the Generalised Gamma lifetime distribution (Robinson, 1998) and to a wide range of lifetime distributions.

\section{Research Results}

In Aboura and Agbinya (2013), several optimization models were studied. For example in their Constant Cost model, there is no dollar value associated with the failure of an

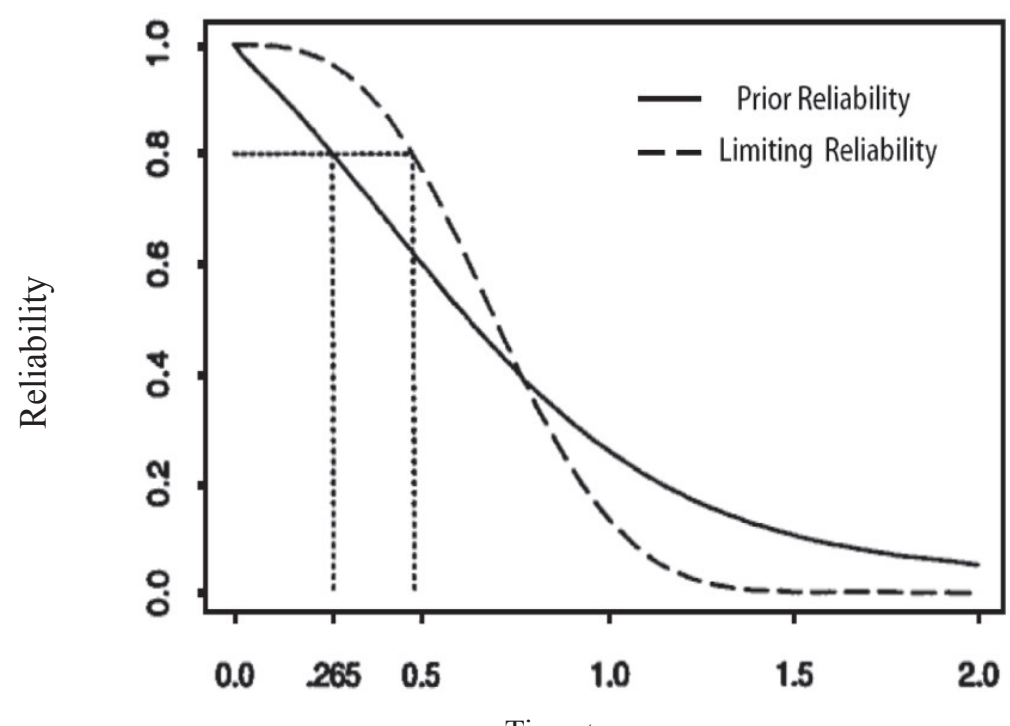

Time $t$

Figure 3: The first replacement time $\Delta T_{1}=0.265$ and the optimal interval $\Delta T_{\infty}=0.481$ 
item. The range of possible time intervals before the next replacement is limited by a constraint on the expected number of failures in the considered interval. The maintenance optimization problem is solved for both collection data protocols and simulation is conducted to show the convergence of the solution. In a simulated example where $\lambda=2$, $\beta=3$ and $K / M=0.2$ they assume that reliability estimates $r^{(5)}=(.99, .95, .60, .05, .01)$ for mission times $t^{(5)}=(.2, .4$, $.75,1,1.2)$ are given by an expert or taken from some other knowledgeable source. $\mathrm{M}$ is the number of operating items and $\mathrm{K}$ is a constraint on the number of failed items (see Aboura and Agbinya, 2013). The optimal value for the first replacement time $T_{1}$ is obtained as the time $t$ at which the prior reliability $R\left(t / D_{0}\right)$ is equal to $1-K / M=0.8$. Therefore in the example the first replacement of all items is to occur at time $T_{1}=\Delta T_{1}=0.265$. The prior reliability function $t / D_{0}$ ) is shown in Figure 3 with the resulting optimal first time interval $\Delta T_{1}=0.265$. The dashed line function in the graph of Figure 3 is $e^{-2 t^{3}}$ with the corresponding limiting optimal time interval $\Delta T_{\infty}=(-\ln (1-.2) / 2)^{1 / 3}=0.481$.

At the successive times $T_{i-1}, i=2,3, \ldots$ the optimal time intervals $\Delta T_{i}$ are obtained as the solution of $R\left(\Delta T_{1} / D_{i-1}\right)=1-K / M$. In the case of complete data, $R\left(\Delta T_{1} / D_{i-1}\right)$ obtains in a closed form while it must be computed numerically in the case of interval censored data. The optimal time intervals between replacements, $\Delta T_{i}$, $i=1,2 \ldots, 20$, are plotted in Figure 4 for a 20 stages simulation of the maintenance routine. The exact failure times are recorded between the replacement times. The horizontal dashed line in Figure 4 marks the limiting optimal time interval $\Delta T_{\infty}=0.481$. As data is gathered between the replacement times, the optimal time intervals improve to finally stabilize around the limiting value.

Although Aboura and Robinson (2013) provide an accurate approach for implementing the maintenance optimization procedure, a simple and practical approach can be substituted that makes use of Maximum Likelihood Estimates at each planned replacement stage. If initial expert information is available in the form of reliability estimates such as $\left(r^{(n)}, t^{(n)}\right)$, Least Squares Estimates can be used to produce the first replacement time $T_{1}$. Maximum Likelihood Estimates will then be used at the next stages for producing the optimal planned replacement times. The MLE approach is attractive in that it eliminates the need for averaging over a prior/posterior distribution, reducing the number of times the renewal function is computed. In an example, Figure 5 shows optimal replacement time intervals $\Delta T_{i}$ for $i=1, \ldots$, 20 as dictated by the MLE based maintenance optimization procedure (Aboura and Robinson, 2013). In this example, the limiting optimal replacement time interval is 0.452 . In their study, Aboura and Robinson (2013) show the behaviour of the optimal replacement time interval $\Delta T_{i}, i=1,2$, ..., 11, for 100 simulated replications. The first replacement time interval $\Delta T_{1}$ is set arbitrarily to 1 . They also display the boxplot of the 10 stages and plot the average optimal replacement time interval, the average taken over the 100 replications. They also compare the average optimal replacement time interval over 10 stages, stage 2 to stage 11 , for the case of 10 and 20 items operating independently. The average of the replacement time interval is taken over 100 simulated replications for the first case (10 items) while

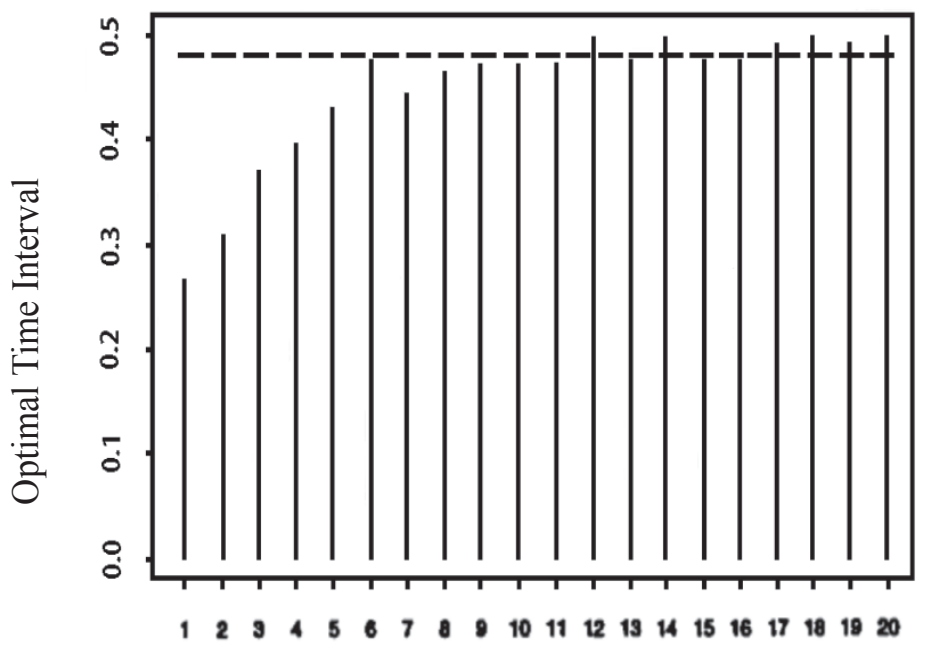

Replacement Stage

Figure 4: The optimal time intervals $\Delta T_{i}, i=1,2 \ldots, 20$ 


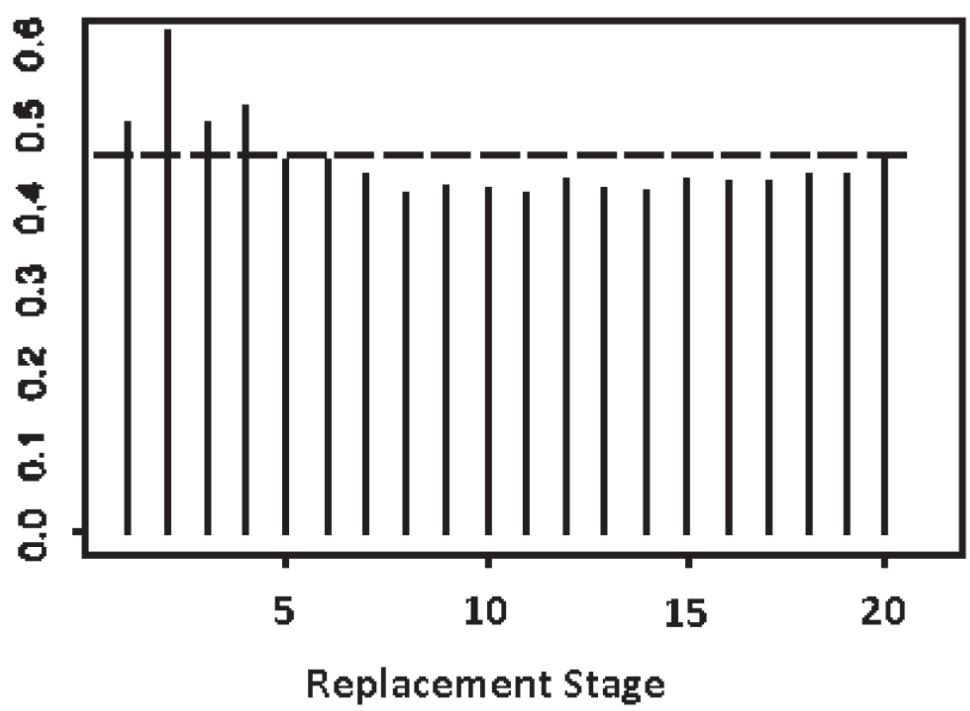

Figure 5: Optimal replacement intervals (Aboura and Robinson, 2013)

it is taken over 50 simulated replications for the second case (20 items).

\section{Discussion}

A variety of optimization models where replacement takes place require statistical modeling and accurate resolution of the optimization problems that ensue. Once these issues are addressed, effective decision support systems can be developed. Adaptive procedures for the optimal replacement of identical items operating under similar conditions are outlined. In Aboura and Agbinya (2013), two data collection scenarios are considered. The procedure was demonstrated and its convergence shown in both data collection cases in simulated examples. The procedure is easy to implement and can result in substantial savings. The adaptive nature of the procedure is a modern feature that permits an updating of the lengths of times between replacements as failure information is gathered. The methodology was developed following technical discussions with an electricity company. Of particular importance in some maintenance scenarios, is the resolution of problems such as the computation of the renewal function. Robinson (1997) solved the Weibull problem. The solution was extended to the Generalized Gamma distribution through the result of Robinson (1998). A whole range of optimization models can be established on the basis of these results. Aboura and Robinson (2013) demonstrate the accurate application of probabilistic updating to the maintenance optimization problems. To avoid excessive computations, the Maximum Likelihood Estimates can be used, as illustrated through simulation.

\section{References}

Abernethy, R. B. (2006). The New Weibull Handbook, Fifth Edition. North Palm Beach, Florida: Published and distributed by Robert B. Abernethy.

Aboura, K. \& Soyer, R. (1986). A User's manual for an interactive PC-based procedure for reliability assessment. George Washington University, Institute for Reliability and Risk Analysis. (Technical Report GWU/IRRA/Serial TR-86/14)

Aboura, K., Singpurwalla, N. D., \& Soyer, R. (1989a). Intelligent systems for reliability. In Proceedings of the 3rd IEEE International Symposium on Intelligent Control, 24-26 Aug. 1988, (103-107), http://dx.doi.org/10.1109/ISIC.1988.65413

Aboura, K., Singpurwalla, N. D., \& Soyer, R. (1989b). Using expert opinion in reliability assessment. Reliability Review, 9(4), 11-12. Retrieved from http://asq.org/qic/displayitem/?item $=3616$

Aboura, K., Singpurwalla, N. D., \& Soyer, R. (1989c). Software for use of expert opinion in reliability. ASQC Quality Congress Transactions, 527-532. Retrieved from http://asq. org/qic/display-item/?item=3616

Aboura, K., \& Campodonico, S. (1992). IPRA, an interactive procedure for reliability assessment incorporating the opinion of one or two experts and failure/survival data: user's manual. George Washington University, Institute for Reliability and Risk Analysis. (Technical Report GWU/IRRA/Serial TR-92/2).

Aboura, K. (1995). Use of informed judgement in the assessment of a prior distribution for the parameters of the Weibull and generalized gamma reliability models. CSIRO. (Report No. DMS-D 95/63).

Aboura, K., \& Agbinya, J. I. (2013). Adaptive maintenance optimization using initial reliability estimates. Journal of Green Engineering. 3, 325-345. 
Aboura, K., \& Robinson, N. I. (2013). Optimal adaptive solutions for maintenance optimization decision support systems. In Proceedings of the 25th International Conference on Systems Research, Informatics and Cybernetics, Advances in Simulation-Based Decision Support and Business Intelligence, 3, (26-30), Baden-Baden, Germany.

Bousquet, N. (2006). A Bayesian analysis of industrial lifetime data with Weibull distributions. Institut National de Recherche en Informatique et en Automatique. (RR-6025). Retrieved January 2014 from http://hal.inria.fr/docs/00/11/57/52/PDF/ RR-6025.pdf

Bousquet, N. (2010). Elicitation of Weibull priors. arXiv:1007.4740 [stat.ME]. Retrieved January 2014 from http://arxiv.org/ abs/1007.4740v3

Constantine, A. G., \& Robinson, N. I. (1997). The Weibull renewal function for moderate to large arguments. Computational Statistics \& Data Analysis, 24(1), 9-27, http://dx.doi. org/10.1016/S0167-9473(96)00052-7

Cox, D. R. (1962). Renewal Theory. London: Chapman-Hall

Fréchet, M. (1927). Sur la loi de probabilité de l'écart maximum. Annales de la Société Polonaise de Mathematique, Cracovie, 6, 93-116.

Goel, P. K. (1988). Software for Bayesian analysis: Current status and additional need. In J. M. Bernardo, M. H. Degroot, D. V. Lindley \& A. F. M. Smith (Eds.), Bayesian Statistics 3 (173-188). Oxford: Oxford Univ. Press.

Kaminskiy, M. P. \& Krivtsov, V. V. (2005). A simple procedure for Bayesian estimation of the Weibull distribution. IEEE Transactions on Reliability, 54(4), 612-616, http://dx.doi. org/10.1109/TR.2005.858093

Mazzuchi, T. A., \& Soyer, R. (1996). A Bayesian perspective on some replacement strategies. Reliability Engineering and System Safety. 51(3), 295-303, http://dx.doi. org/10.1016/0951-8320(95)00077-1

Ouchi, F. (2004). A Literature review on the use of expert opinion in probabilistic risk analysis. World Bank. (Policy Research Working Paper 3201, February 2004). Retrieved January 2013 from http://econ.worldbank.org

Pak, A., Parham, G. A., \& Saraj, M. (2013). Inference for the Weibull distribution based on fuzzy data. Revista Colombiana de Estadística. 36(2), 339-358. Retrieved January 2014 from http://www.emis.de/journals/RCE/V36/v36n2a10PakParhamSaraj1.pdf

ReliaSoft Corporation (2014). Life data analysis (Weibull analysis), an overview of basic concepts. Retrieved January 2014 from http://www.weibull.com/basics/lifedata.htm

Rinne, H. (2009). The Weibull Distribution A Handbook. New York: CRC Press, Taylor \& Francis Group.

Robinson, N. I. (1997). Renewal functions as series. Communications in Statistics. Stochastic Models, 13(3), 577-604, http://dx.doi.org/10.1080/15326349708807440

Robinson, N. I. (1998). Residue series for the generalised gamma renewal function. In J. Noye, M. Teubner, \& A. Gill (Eds.), Computational Techniques and Applications: CTAC97 (591598). Singapore : World Scientific.

Rosin, P., \& Rammler, E. (1933). The laws governing the fineness of powdered coal. Journal of the Institute of Fuel, 7, 29-36.

Singpurwalla, N. D. (1988). An interactive PC-based procedure for reliability assessment incorporating expert opinion and survival data. Journal of the American Statistical Association,
83(401), 43-51, http://dx.doi.org/10.1080/01621459.1988.1 0478563

Singpurwalla, N. D., \& Song, M. S. (1988). Reliability analysis using Weibull lifetime data and expert opinion. IEEE Transactions on Reliability, 37(3), 340-347, http://dx.doi. org/10.1109/24.3765

Singpurwalla, N. D. (2006). Reliability and Risk. A Bayesian Perspective. Chichester, West Sussex: John Wiley and Sons.

Smeitink, E., \& Dekker, R. (1990). A simple approximation to the renewal function. IEEE Transactions on Reliability, 39(1), 71-75, http://dx.doi.org/10.1109/24.52614

Smith, W. L., \& Leadbetter, M. R. (1963). On the renewal function for the Weibull distribution. Technometrics, 5(3), 393-396 http://dx.doi.org/10.1080/00401706.1963.10490107

Soland, R. M. (1969). Bayesian analysis of the weibull process with unknown scale and shape parameters. IEEE Transactions on Reliability, 18, 181-184, http://dx.doi.org/10.1109/ TR.1969.5216348

Sultan, K. S., \& Mahmoud, M. R. (2007). Estimation of parameters of the Weibull distribution based on progressively censored data. International Mathematical Forum, 2(41), 2031-2043.

Weibull, W. (1939). A statistical theory of the strength of material. Proceedings of the Royal Swedish Institute for Engineering Research, 151(1), 1-45.

Weibull, W. (1951). A statistical distribution function of wide applicability. Journal of Applied Mechanics, 18, 293-297.

Weibull, G. W. (1981). Weibull, A statistical distribution function of wide applicability. This Week's Citation Classic. 10, 18-18. Retrieved January 2013, from http:/garfield.library.upenn. edu/classics1981/A1981LD32400001.pdf

Khalid Aboura teaches quantitative methods at the College of Business Administration, University of Dammam, Saudi Arabia. Khalid Aboura spent several years involved in academic research at the George Washington University, Washington D.C, U.S.A. He worked as a Research Scientist at the Commonwealth Scientific and Industrial Research Organization of Australia and conducted research at the University of Technology Sydney. Khalid Aboura was a Scientist at the Kuang-Chi Institute of Advanced Technology of Shenzhen, China.

Johnson I. Agbinya is an Associate Professor in the department of electronic engineering at $\mathrm{La}$ Trobe University, Melbourne, Australia. $\mathrm{He}$ is Honorary Professor at the University of Witwatersrand, South Africa, Extraordinary Professor at the University of the Western Cape, Cape Town and the Tshwane University of Technology, Pretoria, South Africa. His research activities cover remote sensing, Internet of things, biomonitoring systems, wireless power transfer, mobile communications and biometrics systems.

Ali Eskandarian is a theoretical physicist and Dean of the College of Professional Studies \& Virginia Science and Technology Campus at the George Washington 
University, Washington, D.C, U.S.A. He previously held the Distinguished Oliver Professorship in the Integrated
Science and Technology Department at the James Madison University. 